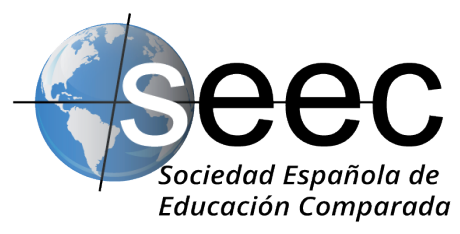

\title{
El derecho a la educación: definiciones constitucionales comparadas en América del Sur
}

\author{
The Right to Education: Comparative \\ Constitutional Definitions in South America
}

\section{Guillermo Ramón Ruiz*}

DOI: $10.5944 /$ reec.39.2021.29247

\section{Recibido: 23 de diciembre de 2010 Aceptado: 18 de febrero de 2021}

\footnotetext{
* Guillermo Ramón Ruz: Doctor en Ciencias de la Educación, Universidad de Buenos Aires (UBA); Master of Arts in Education, University of California Los Angeles (UCLA). Profesor Titular Regular en las Facultades de Derecho y de Psicología de la UBA. Investigador del Consejo Nacional de Investigaciones Científicas y Técnicas (CONICET). Director de proyectos I+D de la Agencia Nacional de Promoción de la Investigación, el Desarrollo Tecnológico y la Innovación; integra el Grupo de Investigación sobre Políticas Educativas Supranacionales de la Universidad Autónoma de Madrid. Sus actividades de investigación refieren al debate pedagógico sobre el contenido material del derecho a la educación, a las reformas educativas de la educación secundaria, y a las políticas de formación de profesorado en perspectiva internacional y comparada. Es autor de libros y artículos científicos de su especialidad. Datos de contacto: E-mail: gruiz@derecho.uba.ar
} 


\title{
Resumen
}

INTRODUCCIÓN. El análisis del derecho a la educación en contextos como América del Sur puede adoptar diferentes enfoques dado que se trata de una región que, por un lado, ha legislado profusamente en materia educativa y, por otro lado, ha experimentado recurrentes procesos de reformas de sus sistemas escolares en las últimas décadas. MÉTODO. En este artículo se analizan las cláusulas constitucionales sobre el derecho a la educación en cuatro países que integran el Mercado Común del Sur (MERCOSUR): Argentina, Brasil, Uruguay (tres de los países miembros) y Chile (uno de los Estados asociados). Se presenta un análisis de las definiciones constitucionales sobre el derecho a la educación en cada uno de los países seleccionados, a la luz de las definiciones conceptuales que tiene este derecho humano. El análisis de las bases constitucionales de la educación permite estudiar de forma comparativa los significados que se le otorgan a este derecho en cada caso. RESULTADOS. En primer lugar, se realiza una definición de este concepto. En segundo lugar, se analizan las constituciones nacionales de cada país para identificar el contenido de este derecho conforme a los respectivos textos constitucionales. DISCUSIÓN. Se realiza una discusión comparativa de las definiciones normativas que sobre el derecho a la educación se evidencian. Finalmente, se plantean los alcances que los textos constitucionales de estos países tienen sobre el derecho a la educación. Ello presenta líneas para interpretar los procesos de reformas escolares, que se han ejecutado durante las últimas décadas, y que han tenido al derecho a la educación como objeto de regulación, lo cual admite pensar en la potencial conformación de un laboratorio normativo en estos países emergentes.

Palabras clave: derecho a la educación; América del Sur; constitucionalismo; política educativa; normativas educativas

\begin{abstract}
INTRODUCTION. Analyzing the right to education in regions such as South America could be done from different perspectives since this region has had several and profound educational legislation, and also because it has had recurrent educational reforms in the last decades. METHODS. In this piece constitutional definitions -regarding the right to education- are taken into account. Four countries of the Southern Common Market (MERCOSUR) are selected: Argentina, Brazil, Uruguay (three of the five full members) and Chile (an associate State). Data for this research has been collected from the constitutional texts of the studied countries. To analyze the data, it was documenting the case of each country, and its constitutional definitions regarding the right to education by drawing upon excerpts field notes. RESULTS. A conceptual definition of the right to education -from the human rights-based perspective- is provided in order to analyze, from a comparative standpoint, the constitutional norms of each country. DISCUSSION. A comparative analysis is made in function to the normative definitions that these four countries have regarding this matter: the right to education. The constrains and the challenges of the national Constitutions are identified, which allow us to find several interpretations of the schooling reforms that these countries have made in the last decades. In all of them the right to education was object of regulations, which admit designing a potential normative laboratory in these emerging countries.
\end{abstract}

Keywords: Right to education; South America; Constitutionalism; Education Politics; Educational Rules 


\section{Introducción}

El análisis del derecho a la educación en contextos como América del Sur puede adoptar diferentes enfoques dado que se trata de una región que, por un lado, ha legislado de forma profusa en materia educativa y, por otro lado, ha experimentado importantes procesos de reformas de sus sistemas escolares. La circulación internacional de discursos político-educativos ha caracterizado la historia de los sistemas escolares desde su conformación en el siglo XIX. Su incremento en las últimas décadas no debería asombrarnos, aunque da cuenta de la propia extensión del tejido educativo en las sociedades contemporáneas (Schulte, 2016) ya que la circulación de propuestas de reformas educativas afecta no solo a los niveles obligatorios de los sistemas, sino que incluye intentos de armonización regional de políticas como las referidas a algunas carreras universitarias y a la formación de profesorado.

En el presente artículo se analizan las cláusulas constitucionales sobre el derecho a la educación en cuatro países que integran el Mercado Común del Sur (MERCOSUR): Argentina, Brasil, Uruguay (países miembros) y Chile (Estado asociado). El análisis de las bases constitucionales de la educación permite estudiar de forma comparativa los significados que se le otorgan a este derecho en cada caso. En general, un texto constitucional se organiza en una parte dogmática, donde se establecen derechos y garantías, y otra parte orgánica, donde se diagrama la forma del Estado y la modalidad de distribución jurídica territorial del poder del Estado. Ello a su vez permite identificar, por un lado, el contenido del derecho a la educación y, por otro, la distribución de competencias en materia educativa entre los distintos poderes y niveles de gobierno que existen en un Estado. Este trabajo se concentra solamente en el primero de estos dos aspectos, y utiliza al MERCOSUR como criterio de demarcación del ámbito geográfico y de los países seleccionados.

En las últimas décadas los países del sur del continente americano han experimentado recurrentes reformas escolares las cuales, al estar enmarcadas en definiciones normativas, han regulado al derecho a la educación como premisa para la superación de desigualdades sociales. En este artículo se presenta un análisis de las definiciones constitucionales sobre el derecho a la educación en cada uno de los países seleccionados. En primer lugar, se realiza una definición de este concepto. En segundo lugar, se analizan las constituciones nacionales de cada país para identificar el contenido de este derecho conforme a los respectivos textos constitucionales de países que integran un bloque regional en proceso de integración. Posteriormente, se realiza una discusión comparativa de las disposiciones normativas que sobre el derecho a la educación que se evidencian. Finalmente, se plantean los alcances y limitaciones que los textos constitucionales de estos países tienen sobre el derecho a la educación, lo cual presenta líneas para interpretar los procesos de reformas escolares que se han ejecutado durante las últimas décadas y que han tenido al derecho a la educación como objeto de regulación.

\section{Materiales y métodos}

En contextos contemporáneos de reformas educativas cabría prestar atención a cómo inciden las nuevas realidades espaciales en la transferencia internacionales de discursos y políticas de reforma. Ciertamente dichas realidades espaciales mantienen, pero también desbordan, al Estado-nación como eje analítico central para el estudio de la 
transferencia. Las dimensiones espaciales en las cuales operan los sistemas y las políticas educativas revelan nuevos modos en los que se gobierna la educación formal y su organización en contextos geográficos con múltiples movilidades educativas. Durante las últimas cuatro décadas se ha desarrollado una serie de estudios de lógica global que ya no se concentran sobre instituciones educativas de distintos Estados nacionales, o sociedades, o culturas sino sobre los procesos y las transformaciones educativas inherentemente trasnacionales y que tendrían un carácter sistémico mundial (Schriewer, 2014; Steiner-Khamsi, 2010).

En términos metodológicos, el diseño de este estudio incluye técnicas cualitativas entre las que se destacan el análisis de contenido de documentos normativos, la generación de tipificaciones y la formulación de grillas comparativas de las fuentes provenientes de los países considerados. La reconstrucción teórica dio lugar a una hermenéutica que se realiza desde formatos de análisis críticos de los corpus discursivos que constituyen las fuentes constitucionales estudiadas. Según Schriewer (2010), la comparación como metodología es teóricamente dependiente por lo que en general adopta diseños diversos en función de las conceptualizaciones metateóricas que refieren a la causalidad, a la/s teoría/s y a las capacidades explicativas de cada encuadre. En el caso en particular de la comparación de países, la educación comparada tendría que realizar la lectura de los contextos en los cuales las prácticas y normas educativas se producen y desarrollan (Broadfoot, 2000).

En este artículo se trabaja con unidades de análisis similares, pero no idénticas ya que se trata de un grupo de textos constitucionales de países que, si bien comparten un contexto regional y antecedentes históricos comunes, constituyen unidades políticas independientes, con desarrollos específicos en materia de las definiciones normativas sobre el derecho a la educación. Todos ellos integran un espacio regional (MERCOSUR) que no solo aspira a una unión aduanera sino también política, al menos en el largo plazo, la cual incluye una dimensión educativa, y la potencial conformación de un laboratorio normativo. En consecuencia, en este trabajo la comparación transnacional se focaliza en uno de los conceptos básicos que organiza la política educativa: el derecho a la educación en función de su contenido conforme a las Constituciones nacionales analizadas dentro del ámbito geográfico demarcado por el MERCOSUR. Desde esta perspectiva, se interpreta que el estudio de la transferencia de políticas constituye un terreno fértil para comprender los intereses contradictorios que afectan a las políticas educativas en contextos específicos (Steiner-Khamsi, 2016). Este enfoque favorece, a su vez, la identificación de un proceso de diseminación en la transferencia de políticas educativas (Dale, 1999), al menos en el plano de las cláusulas constitucionales de los países estudiados, al identificar los alcances que posee la regulación del derecho a la educación en cada Constitución nacional.

\section{Resultados}

\subsection{El concepto del derecho a la educación}

En los últimos siglos el derecho a la educación se ha constituido en uno de los elementos de las transferencias internacionales. Primero como proclama igualadora de la población, y luego como concepto de las ciencias jurídicas que refiere a uno de los derechos humanos fundamentales. Ha sido incorporado con centralidad incomparable en la agenda 
pública, y en los programas de gobiernos de todas las orientaciones ideológicas. Asimismo, concitó acuerdos internacionales que se tradujeron en programas de acción de alcance global y compromisos por parte de los Estados para garantizarlo y promoverlo. Se considera que es un derecho social, que es un derecho humano, que es un derecho fundamental. ${ }^{1}$ La educación como derecho ocupa actualmente un rol destacado en su reconocimiento internacional como derecho humano y también en el interior de los Estados. Sin embargo, a poco que se realiza un análisis más detallado de sus itinerarios históricos, así como de las definiciones constitucionales existentes, se observan particularidades que merecen un estudio singular acerca de las condiciones de vigencia del derecho a la educación a fin de evitar incurrir en generalizaciones que no se avienen con los resultados que ofrecen las acciones del Estado (Ruiz, 2020).

En función del enfoque metodológico previamente mencionado, es posible notar la importancia del reconocimiento de la educación como un derecho humano fundamental, con múltiples contenidos que imponen obligaciones concretas a cargo de los Estados nacionales. El contenido de este derecho merece ser estudiada a la luz de los desarrollos normativos supraestatales y locales, así como de sus políticas, en una línea histórica contemporánea que permita reconocer sus alcances y limitaciones. Con este encuadre, en este artículo se presenta un estudio que incluye la descripción analítica de las definiciones constitucionales, de cada uno de los Estados seleccionados, para comprender la regulación contemporánea del derecho a la educación.

\begin{abstract}
1 Los derechos de prestación, entendidos como derechos sociales o derechos a acciones fácticas positivas, generan obligaciones de dar. Se alude aquí a la obligación de cumplir, a la necesidad de que el Estado garantice el derecho a la educación en su sistema político y en su ordenamiento jurídico nacional y que arbitre los medios materiales para poder asegurar su ejercicio efectivo. En este sentido, este tipo de obligación requiere que el Estado adopte medidas positivas que permitan dar plena efectividad y ejercicio de este derecho, especialmente a individuos o grupos que no estén en condiciones (por razones ajenas a su voluntad) de ejercer dicho derecho por sí mismos. Consecuentemente, desde este enfoque, es susceptible incorporar el mayor caudal de obligaciones que del Estado se derivan para con la educación como derecho social. En especial, desde esta perspectiva aparece con claridad la obligación indelegable y de inversión prioritaria de la autoridad estatal para garantizar ese derecho con acciones positivas. Los derechos sociales en su conjunto representan al constitucionalismo social, en el cual se plasma la lucha por el reconocimiento de diversos grupos o colectivos sociales. El Estado dentro de esta perspectiva es intervencionista en lo económico (capital) y en lo social (trabajo) y aparece como mediador de los conflictos de clases. Con el constitucionalismo social, la contradicción, la desigualdad y la diferencia, entran en la Constitución (Elster, 1998).
\end{abstract}

En relación con la definición de los derechos fundamentales resultad sustantiva, entre otras, la obra de R. Alexy (2008) y una de sus discípulas, L. Clérico (2009). En particular Clérico propone, sobre la base del planteo de Alexy, la reconstrucción de los contenidos del derecho en términos de obligaciones impostergables o inmediatas, por un lado, y de cumplimiento progresivo a cargo del Estado, por el otro, y plantea -asimismoel examen de proporcionalidad como un modo de resolver eventuales o posibles conflictos de derechos. La noción del derecho fundamental toma de la definición de derechos humanos su carácter de derechos universales y abstractos que son reconocidos por igual para todas las personas, más allá de su ciudadanía nacional (Scioscioli, 2015). Los principios básicos que definen a los derechos humanos son: universalidad e inalienabilidad; indivisibilidad e interdependencia; integralidad (diversidad de derechos, integrados en una totalidad sin jerarquías o valores internos); progresividad (el reconocimiento de los alcances del derecho ya garantizados es irreversible).

Lo sustantivo de este planteo es que la educación es pensada y definida como un derecho con un amplio reconocimiento a partir del desarrollo del internacional de los derechos humanos. Ello ha implicado que contase con un consenso significativo por parte de los Estados y se ha visto reflejado además en la inclusión del derecho a la educación en los cinco instrumentos internacionales de derechos humanos más importantes surgidos de la Declaración Universal de los Derechos Humanos de 1948 (Convención sobre la eliminación de todas las formas de discriminación racial, de 1965; Pacto internacional de derechos civiles y políticos, de 1966; Pacto internacional de derechos económicos, sociales y culturales, de 1966; Convención sobre la eliminación de todas las formas de discriminación contra la mujer, de 1979; Convención sobre los derechos del niño, de 1989). 
Un punto de demarcación importante en lo que atañe a la configuración del derecho a la educación, estuvo dado por la conformación del derecho internacional de los derechos humanos en el contexto de finalización de la Segunda Guerra Mundial. En el ámbito europeo, la mayoría de los países occidentales aprobaron constituciones que reflejaron los cambios económicos y sociales ocurridos en las décadas previas, así como las reacciones a las formas fascistas de organización de los Estados de las décadas precedentes. Según Ferrajoli (2016), en países como Italia, Alemania, España y Portugal que atravesaron la experiencia del fascismo, se aprobaron constituciones rígidas en el plano jurídico, es decir, caracterizadas por mayor fuerza normativa y garantista. Dicho constitucionalismo rígido permitió constitucionalizar no solo los derechos de libertad, los derechos civiles, sino además los derechos sociales como derechos fundamentales. El garantismo es un rasgo específico del constitucionalismo del siglo XX. Las garantías son prohibiciones y obligaciones presentes en los derechos constitucionalmente establecidos: los derechos de libertad suponen un paso atrás para el Estado, dado que existen prohibiciones para intervenir en la esfera de las libertades que garantizan la autodeterminación individual; por su parte, los derechos sociales implican un paso adelante, en tanto obligan al Estado a intervenir en la vida de los ciudadanos para garantizar las correspondientes prestaciones sociales (Ferrajoli, 2016). Todo ello daba cuenta de una transformación en la forma del Estado, que pasaba de ser básicamente protector-represor a ser cada vez más promocional: el Estado no solo debía impedir, sino que debía promover; no resultaba suficiente la visión de la justicia conmutativa -basada en el criterio de la igualdad formal-, sino que debía promoverse la justicia distributiva. La gran transformación del siglo XIX exigió que el Estado asumiera responsabilidades frente a la urbanización, la industrialización y la proletarización de la sociedad. En tal sentido, la institucionalización de la educación escolar a través de sistemas masivos constituyó una de esas respuestas, que alcanzó una escala global luego de la Segunda Posguerra (Ruiz, 2020).

Ahora bien, el contenido del derecho a la educación es un tema de alta relevancia para la agenda pública. En primer lugar, hay que señalar que existen obligaciones estatales para garantizar la educación como derecho humano fundamental. La vigencia de este derecho se evidencia en las políticas públicas que los Estados implementan para promover la escolarización masiva de la población. Se puede pensar que los sistemas nacionales de escolarización constituyen el indicador más notorio de este derecho humano y la expansión, el acceso y los alcances de la educación escolar conforman proclamas igualadoras sumamente consensuadas en las sociedades, por encima de las diferencias sociales, ideológicas y culturales. En segundo lugar, cabe destacar que la Oficina del Alto Comisionado para los Derechos Humanos de las Naciones Unidas (2006) señaló que el derecho internacional concerniente a los derechos humanos se ha elaborado con el propósito de ampararlos y hacerlos efectivos para que las personas puedan vivir una vida plena, libre, segura y sana. ${ }^{2}$ Bajo este marco, el derecho a la educación ha ganado terreno

2 En el ámbito internacional, los esfuerzos por hacer operativos los derechos humanos -en el marco de las políticas públicas- han llevado a que la conceptualización de tales derechos vuelva a redefinirse ya no solo como nueva ética y moral tendentes a generar «un cambio cultural basado en el reconocimiento recíproco, sino también como metas políticas y programáticas que permitan el seguimiento y la evolución efectiva de dicho cambio». Con estas palabras, la Oficina del Alto Comisionado para los Derechos Humanos definió al enfoque de derechos como marco conceptual para el proceso de desarrollo humano que desde el punto de vista normativo está basado en normas internacionales de derechos humanos y desde el punto de vista operacional está orientado a la promoción y protección de los derechos humanos. Esta noción se ha visto reforzada con las bases sentadas en la «Declaración de Entendimiento Común» (2003) que refiere al 
en su reconocimiento y alcances en el contenido de varios instrumentos. No obstante, cuando se observa cómo es pensado el derecho a la educación se nota la invocación de ideas muy diversas que concitan la atención de todo el arco ideológico y que posibilitan la formulación de demandas -referidas a este derecho- que aparecen como satisfactorias para diferentes ámbitos y públicos (Ruiz, 2020).

En este trabajo se define a la educación desde el enfoque de derechos humanos, lo cual permite pensarla como un derecho social, cultural, político, económico. Se podría decir que el derecho a la educación es super-derecho que, si bien tiene un amplio reconocimiento internacional por ser uno los derechos humanos fundamentales, y por generar obligaciones a los Estados para garantizarlo, no logra ser ejercido de manera igualitaria por la población, a pesar de ser el más igualador de los derechos humanos. Para definirlo se podría tomar como punto de partida que todas las personas tienen derecho a la educación debido a que las capacidades para interpretar el mundo y actuar en él resultan esenciales para la vida humana (Ruiz, 2020). Al considerar a todas las personas, se subraya el principio de igualdad, el cual se encuentra en la base de los derechos humanos fundamentales. Congruente con esta definición se encuentra la teoría de las capacidades (Sen, 2011) que enfatiza la noción de autonomía del individuo respecto de sus condiciones de vida y de las desigualdades estructurales -económicas y culturales, entre otras-, para permitirle a la persona participar de manera significativa en el aprendizaje (McCowan, 2013) y convertir ese aprendizaje en oportunidades reales para el desarrollo humano en función de aquello que se valora y que resulta significativo para su vida.

El derecho a la educación a su vez comprende desde el plexo normativo internacional un importante catálogo de obligaciones a cargo de los Estados nacionales: obligación de respetar, de proteger, de cumplir, de realizar y de garantizar la educación, la cual a la vez no solo es uno de los derechos humanos fundamentales, sino que tiene implicancias para el ejercicio de los demás derechos humanos (Ruiz, 2020). A la luz del derecho internacional de los derechos humanos, el derecho a la educación supone:

- un conjunto de definiciones relativas a los objetivos y fines de la educación

- una serie de obligaciones a cargo del Estado para asegurar el acceso a la educación mediante la promoción de la gratuidad y otras herramientas

- el respeto del derecho de los particulares a fundar escuelas diferentes a las del Estado y el derecho de los padres a decidir la orientación de la educación que recibirán sus hijos; todo ello está contemplado en las definiciones constitucionales de cada país.

En función del análisis realizado para este estudio, se distinguen los casos de Argentina y de Brasil debido a que ambos poseen regímenes federales de gobierno; lo cual conlleva implicancias para la distribución de competencias entre los diferentes niveles -federal, estadual/provincial y municipal-, principalmente para analizar las obligaciones del Estado en materia del derecho a la educación a la luz de la coordinación prevista en el plano de las normas de estos países (tema que queda fuera de este artículo). En los demás casos estudiados, el reconocimiento y la regulación del derecho a la educación tienen diferentes niveles de complejidad en función de cómo han sido incorporados los estándares internacionales sobre derechos humanos en los textos constitucionales, lo cual obedece a razones históricas y al período en el cual fue aprobada la Constitución nacional actualmente vigente en cada caso.

enfoque basado en los derechos humanos, aplicado a la cooperación y a los programas de desarrollo de las Naciones Unidas. 


\subsection{Las definiciones constitucionales sobre el contenido del derecho a la educación en Argentina}

La Constitución argentina es la más antigua de los casos seleccionados y prevé un amplio reconocimiento de la educación como derecho; el cual es objeto de regulación en diferentes cláusulas, algunas ya previstas en la Constitución histórica de 1853-1860, y otras que han sido incorporadas como resultado de su reforma en el año $1994 .{ }^{3}$ Las cláusulas que refieren al contenido del derecho a la educación son las siguientes:

- en el artículo 14 se reconoce a todos los habitantes el derecho de enseñar y aprender

- en el artículo 20, este derecho se extiende específicamente a los extranjeros

- en el inciso 22 del artículo 75, se incluyen con jerarquía constitucional los instrumentos internacionales de derechos humanos que poseen disposiciones relativas al derecho a la educación

- los incisos 17 y 23 del artículo 75 demarcan los alcances que posee el contenido del derecho a la educación y las medidas de acción positiva que desde la legislación nacional deben promover la igualdad material y de reconocimiento

Si se diferencia entre los objetivos y fines de este derecho, es posible mencionar los siguientes artículos:

- consolidar la unidad nacional con respeto a las particularidades provinciales y locales (artículo 75, inciso19)

- asegurar la participación de la familia y la sociedad (artículo 75, inciso 19)

- promover los valores democráticos (artículo 75, inciso 19)

- promover la igualdad de oportunidades y posibilidades sin discriminación (artículo 75, incisos 19 y 23);

- asegurar la educación intercultural y bilingüe para los pueblos originarios (artículo 75, inciso 17)

- asegurar la educación ambiental (artículo 41)

- asegurar la educación sobre los derechos como consumidores y usuarios de bienes y servicios (artículo 42)

De esta enumeración sobresale el artículo 14 de la Constitución que redactado en 1853 se mantiene vigente y establece que: «Todos los habitantes de la Nación gozan de los siguientes derechos conforme a las leyes que reglamenten su ejercicio, a saber... de enseñar y aprender». Se enuncian aquí dos derechos que aparecen en un plano de igualdad, sin que se establezca una relación jerárquica entre ellos; solo puede pensarse en ella por el hecho de que el derecho a enseñar está colocado en primer lugar. Sin embargo, el análisis de las implicancias de estos derechos enunciados en el artículo 14 da cuenta de la naturaleza diferente de cada uno. Así, si se toma en primer lugar el derecho a enseñar, 3 Esta Constitución fue aprobada en el período de organización institucional del Estado argentino, entre los años 1853 y 1860. Continúa vigente con las reformas introducidas en los años 1866, 1898, 1957 y 1994. Esta última es la más importante ya que introdujo técnicas del constitucionalismo social y del constitucionalismo internacionalizado. Estas definiciones refieren a los movimientos del constitucionalismo: se considera que las constituciones históricamente han avanzado en técnicas para el mejor logro de sus finalidades (Elster, 1998). 
se debería considerar que este implica varias cuestiones y que tiene estrecha relación con la libertad de enseñanza. Por otro lado, a diferencia de la libertad de enseñanza que requiere un corrimiento del Estado, un no hacer por parte del Estado, un paso atrás, el derecho a aprender requiere en cambio una acción positiva del Estado que permita respetarlo, garantizarlo, promoverlo, así como facilitar su ejercicio a todos los habitantes, es decir, un paso adelante. La redacción del artículo 14 no ha sufrido modificaciones en las sucesivas reformas constitucionales. Con la reforma constitucional de 1994, se han incorporado cláusulas que amplían el contenido de este derecho, sobre todo en el artículo 75 que refiere a las competencias del Congreso Nacional (incisos 17, 19, 22 y 23); aunque también se pueden mencionar los artículos 42 y 43 que refieren al contenido derivado del derecho a la educación (Scioscioli, 2015). En consecuencia, es posible sostener que, según esta Constitución Nacional, el derecho a la educación tiene como finalidad la plena realización de la persona en toda su potencialidad y al mismo tiempo es un derecho fundamental que posibilita el efectivo ejercicio de otros derechos, sobre la base del reconocimiento de la diversidad y del principio de igualdad real y material. El Estado argentino reguló intensamente el ejercicio del derecho a la educación en las últimas décadas a través de dos leyes, una en el año 1993 y otra en el año 2006.

\subsection{Las bases constitucionales del derecho a la educación en Brasil}

El derecho a la educación fue reconocido como derecho público y social recién en la Constitución Federal de 1988. Antes de ello, no había una obligación formal para el Estado de garantizarlo y era regulado como una asistencia hacia aquellos que no podían pagar por ella. En efecto, se trataba de un conjunto de preceptos de carácter programáticos que reflejaban buenas intenciones. Así, en la Constitución Imperial de 1824 se estableció, entre los derechos civiles y políticos, la gratuidad de la instrucción primaria para todos los ciudadanos y se previó la creación de colegios y universidades, tal como se prevé en el artículo 179: "A inviolabilidade dos direitos Civis e Políticos dos cidadãos brasileiros, que tem por base a liberdade, é garantida pela Constituição do Império, pela maneira seguinte: [...] XXXII. A instrução primária é gratuita a todos os cidadãos. XXXIII. Collegios, e Universidades, aonde serão ensinados os elementos das Sciencias, Bellas Letras, e Artes.»

Sin embargo, su regulación se traducía fundamentalmente como un medio para garantizar derechos civiles y políticos y su eficacia resultó marcadamente limitada. En efecto, transcurridos 50 años de dicha Constitución, un relevamiento de 1872 reveló que Brasil figuraba entre los países con mayor porcentaje de analfabetismo, con números que oscilaban entre el 78 \% y el 82 \% de la población (Ferraro, 1985). La Constitución Republicana de 1891, por su parte, se dedicó a establecer la competencia legislativa de la Unión y los Estados en materia educacional -artículo 34 inc. 30; artículo 35 incs. $3^{\circ}$ y $4^{\circ}-$, e instauró la enseñanza laica en los establecimientos públicos: «Artículo 72. [...] 6. Será leigo o ensino ministrado nos estabelecimentos públicos». Sin embargo, para observar algún avance significativo en materia de legislación constitucional sobre el derecho a la educación fue necesario esperar a la Constitución de 1934: en ella se incluyó un capítulo específico sobre el tema -Capitulo II: Da Educação e da Cultura- en el cual se afirmaba que la educación «es un derecho de todos y debe ser impartido por la familia y los poderes públicos» (artículo 149). Además, fue estipulada la competencia legislativa de la Unión para trazar directrices de la educación nacional (artículo 150), la libertad de cátedra (artículo 155), la asistencia hacia alumnos carenciados (artículo 157, inciso $2^{\circ}$ ), 
la asignación de cargos de enseñanza mediante concurso público (artículo 158), entre otras disposiciones de relevancia. Aun así, estos avances mantenían en gran medida un carácter declaratorio y una matriz programática ya que no brindaban herramientas de exigibilidad a los ciudadanos para propiciar su eficacia. Las Constituciones siguientes -las de 1937, 1946 y 1967 y sus enmiendas- poco sumaron al derecho a la educación conforme lo previsto en la de 1934.

En la Constitución Federal de Brasil de 1988 el derecho a la educación ha recibido una regulación detallada y minuciosa que además ha incorporado importantes innovaciones respecto a las que le precedieron. De esa manera, pueden encontrarse una serie de artículos entre los que se destacan aquellos referidos a la asignación de competencias legislativas, la organización del sistema, el acceso a la educación, su calidad y financiamiento, la asignación de responsabilidades al Estado y a la familia, la distribución de funciones entre los entes de la federación, la disposición de instrumentos para garantizar su eficacia, entre otros.

El derecho a la educación se encuentra incluido dentro del capítulo de los llamados derechos sociales (artículo $6^{\circ}$ ), esto es, los denominados derechos de segunda generación, en el ámbito de los derechos fundamentales: «São direitos sociais a educação, a saúde, a alimentação, o trabalho, a moradia, o transporte, o lazer, a segurança, a previdência social, a proteção à maternidade e à infância, a assistência aos desamparados, na forma desta Constituição».

A diferencia de los derechos de primera generación, cuya principal preocupación se dirige hacia la defensa de la libertad de los individuos frente al imperium del Estado, y propicia una abstención o no intervención por parte de este último, los de segunda generación, por el contrario, procuran una acción positiva del poder estatal y su principal finalidad se dirige hacia la protección de la igualdad y la dignidad humana, y actúan en forma complementaria a los primeros. Es esta última acepción la que impregna el orden normativo sobre el derecho a la educación en la Constitución Federal de 1988 de Brasil y que significó un cambio sustantivo respecto de las constituciones anteriores (Alvarez et al, 2020).

En el Capítulo III denominado «De la Educación, Cultura y Deporte» perteneciente al Título VIII «Del Orden Social», la CF dedica una sección específica -Sección I- a la educación. Allí se desarrollan los preceptos generales que regulan la materia y que conforman el núcleo central del régimen constitucional sobre el derecho a la educación. En lo que atañe al alcance, objetivos y deberes previstos en la Constitución, se evidencia, en primer término, el artículo 205 determina que la educación es un derecho de todos y un deber del Estado y de la familia. Esto último se encuentra reforzado por lo dispuesto en el artículo 227 en el que se afirma que «es deber de la familia, de la sociedad y del Estado, asegurar al niño, al adolescente y al joven, con absoluta prioridad, el derecho a [...] la educación [...]». Además, el artículo 205 establece que la educación será promovida e incentivada con la colaboración de la sociedad, persiguiendo un triple objetivo, a saber:

- El pleno desarrollo de la persona

- Su preparación para el ejercicio de la ciudadanía

- Su calificación para el trabajo

Luego, la Constitución de 1988 profundiza acerca del rol del Estado en materia educacional y establece que su deber con la educación se hará efectivo mediante la garantía 
de lo detallado en el artículo 208:

- educación básica obligatoria y gratuita desde los 4 (cuatro) a los 17 (diecisiete) años de edad

- progresiva universalización de la enseñanza secundaria gratuita

- atención educativa especializada para personas con discapacidad

- educación infantil, en guarderías y jardín de infantes a los niños de hasta 5 (cinco) años de edad

- acceso a los niveles más elevados de enseñanza, investigación y creación artística

- oferta de enseñanza nocturna regular

- atención al educando, en todas las etapas de la educación básica, por medio de programas suplementarios de material didáctico escolar, transporte, alimentación y asistencia de salud

Por último, la Constitución reafirma que el acceso a la enseñanza obligatoria y gratuita es un derecho público subjetivo y su no ofrecimiento por el Poder Público. A su vez, la Constitución prescribe ciertos principios -artículo 206-que deberán respetarse en el momento de impartir la enseñanza:

- igualdad de condiciones para el acceso y la permanencia en la escuela

- iibertad de aprender, enseñar, investigar y difundir toda opinión, arte o saber

- pluralismo de ideas y concepciones pedagógicas, y coexistencia de instituciones públicas y privadas de enseñanza

- gratuidad de la enseñanza pública en establecimientos oficiales

- valorización de los profesionales de la educación escolar, a través de planes de carrera y concursos públicos para acceder a las plazas

- piso salarial nacional para los docentes del sector público

Un último aspecto importante está dado en lo que concierne a la relación existente entre los entes federados, la Constitución Federal ha previsto en su Capítulo Sexto algunos casos que podrían dar lugar a la intervención por parte de la Unión o los Estados hacia los niveles inferiores de la administración, como una prerrogativa de excepción, cuando estos últimos no cumplan con alguna de sus obligaciones en materia educacional (artículo 34). La ley del año 1996 regula el ejercicio de este derecho (Cury, 2016).

\subsection{El derecho de la educación en la Constitución chilena aprobada durante la dictadura}

Lo primero a destacar en este país es que el texto constitucional chileno fue aprobado durante la dictadura de Augusto Pinochet; 4 tuvo un plebiscito de ratificación y entró en vigor en marzo de 1990. Es un texto constitucional que experimentó varias enmiendas entre los años 1989 y 2017.5 El derecho a la educación se ubica en la Constitución -apro-

$4 \quad$ El régimen dictatorial del Gral. Pinochet se extendió entre el 11 de septiembre de 1973 y el 11 de marzo de 1990.

$5 \quad$ El 25 de octubre de 2020 se llevó a cabo plebiscito para que la ciudadanía decidiera sobre una 
bada en 1980- como derecho humano, en el capítulo III «De los derechos y deberes constitucionales», artículo 19, inciso 10 que afirma:

«La educación tiene por objeto el pleno desarrollo de la persona en las distintas etapas de su vida. Los padres tienen el derecho preferente y el deber de educar a sus hijos. Corresponderá al Estado otorgar especial protección al ejercicio de este derecho. Para el Estado es obligatorio promover la educación parvularia (de o a 6 años de edad) y garantizar el acceso gratuito y el financiamiento fiscal al segundo nivel de transición sin que este constituya requisito para el ingreso a la educación básica. (...) La educación básica y la educación media son obligatorias, debiendo el Estado financiar un sistema gratuito con tal objeto, destinado a asegurar el acceso a ellas de toda la población. En el caso de la educación media, este sistema, en conformidad a la ley, se extenderá hasta cumplir los 21 años. (...) Corresponderá al Estado, asimismo, fomentar el desarrollo de la educación en todos sus niveles; estimular la investigación científica y tecnológica, la creación artística y la protección e incremento del patrimonio cultural de la Nación. Es deber de la comunidad contribuir al desarrollo y perfeccionamiento de la educación».

El inciso 11 del mismo artículo de la Constitución dispone que:

«La libertad de enseñanza incluye el derecho de abrir, organizar y mantener establecimientos educacionales. La libertad de enseñanza no tiene otras limitaciones que las impuestas por la moral, las buenas costumbres, el orden público y la seguridad nacional. (...) La enseñanza reconocida oficialmente no podrá orientarse a propagar tendencia político partidista alguna. Los padres tienen el derecho de escoger el establecimiento de enseñanza para sus hijos. Una ley orgánica constitucional establecerá los requisitos mínimos que deberán exigirse en cada uno de los niveles de la enseñanza básica y media y señalará las normas objetivas, de general aplicación, que permitan al Estado velar por su cumplimiento. Dicha ley, del mismo modo, establecerá los requisitos para el reconocimiento oficial de los establecimientos educacionales de todo nivel».

Los establecimientos que brindan servicios de educación en Chile se dividen según su dependencia administrativa en:

- Establecimientos estatales: se trata de establecimientos de educación superior que gozan de autonomía académica y administrativa, pero que son financiados por el Estado. Existen también, en el nivel Preescolar, los establecimientos pertenecientes a la Junta Nacional de Jardines Infantiles, entidad pública autónoma que recibe financiamiento estatal

- Municipales: son establecimientos públicos de propiedad y financiamiento principalmente estatal, administrados por las municipalidades del país. Estos presentan servicios de enseñanza Parvularia, Básica y de Enseñanza Media Humanístico-Científica y Técnico-Profesional

reforma constitucional. Como resultado de la consulta se convocó a una Convención Constituyente para redactar una nueva Constitución y reemplazar a la de 1980. Se prevé a la vez un plebiscito ratificatorio en el año 2022. Este proceso no se incluye en este estudio. 
- Particulares subvencionados: son establecimientos de propiedad y administración privada, pero que en el caso de los niveles Preescolar $-2^{\circ}$ nivel de Transición-, Básico y Medio, reciben financiamiento estatal mediante subvención por alumno matriculado y efectivamente asistiendo a clases. En el caso de la Educación Superior, los establecimientos particulares subvencionados reciben diversos aportes del Estado fijados anualmente en el presupuesto nacional

- Particulares pagados: son centros de formación privada, cuya propiedad, administración y financiamiento corresponde a particulares. Ofrecen servicios para todos los niveles del sistema educativo

Es un texto aprobado durante una dictadura militar con variadas enmiendas en los años ulteriores. Si bien se define a la educación como un derecho humano, a la vez se le otorga centralidad a la libertad de enseñanza como prerrogativa para favorecer el rol de los particulares privados en la creación y desarrollo del servicio educativo. Ello se evidencia precisamente en la legislación educativa que se aprobó en este país, tanto durante el período del gobierno militar como aquella de los gobiernos democráticos (la legislación general para el conjunto del sistema escolar más reciente data del año 2009). En dichas leyes (que no son parte de este estudio) se evidencian definiciones sobre los alcances del derecho a la educación: limitaciones en la cuestión de la gratuidad de los estudios en el nivel superior, la desregulación de la educación privada, el acceso a fondos públicos para desarrollo del sector privado. Son notorias, sobre todo en el plano de la legislación educativa (más que en las cláusulas constitucionales) las diferencias del caso chileno en relación con los demás países, ya que su legislación educativa ha dado lugar a reformas escolares desde la década de 1980 que han afectado el alcance del derecho a la educación, sobre todo por favorecer al sector privado en desmedro del público (Santa Cruz Grao y Olmedo, 2012).

\subsection{La Constitución uruguaya a la vanguardia en materia del reconocimiento del derecho a la educación}

La Constitución de la República Oriental del Uruguay de 1967 contiene un amplio marco normativo en materia de educación. No se limita a reconocerla como un derecho fundamental ya que establece una precisa serie de instrucciones organizacionales y derechos y garantías con jerarquía constitucional, desde el establecimiento de la obligatoriedad de la educación primaria y media (artículo 70) y la utilidad pública de la gratuidad (artículo 71), el deber y derecho de los padres a educar a sus hijos (artículo 41), la organización en Consejos Directivos (artículo 202) hasta la autonomía de la Universidad de la República (artículo 175, párrafo $6^{\circ}$ ).

De conformidad con la Constitución, en Uruguay es obligatoria la enseñanza primaria y la enseñanza media, agraria o industrial (artículo 70). Por su parte, el artículo 71 establece la utilidad social de la gratuidad de la enseñanza oficial primaria, media, superior, industrial, artística y física. De su redacción surge la gratuidad de todos los niveles de la educación, y deja abierta la posibilidad a la UDELAR y a la UTEC de cobrar una matrícula a quienes cursen estudios de posgrado (Biasco, 2001). A su vez, se hace extensiva la gratuidad al nivel inicial debido a su obligatoriedad. Por otra parte, el mentado artículo 71 no se limita al derecho/deber de los padres; también garantiza una protección especial para aquellas familias numerosas y manda al legislador a crear una ley que garantice la protección de la infancia y la juventud. 
En materia institucional, es fundamental el artículo 202 de la Constitución. En la práctica, la función de los consejos directivos es llevada a cabo por la Administración Nacional de Educación Pública (ANEP) y la Universidad de la República. La primera para el ámbito privado en todos sus niveles, y público en los niveles inicial, primario, medio, técnica y formación en educación terciaria; la segunda en lo concerniente a la educación terciaria pública y privada excepto la formación en educación. En el mismo orden, el artículo 203 y el 204 establecen que el Congreso debe sancionar leyes que regulen el funcionamiento de estos consejos, y establece como requisito la mayoría absoluta del total de cada cámara.

La Constitución no se limitó al ámbito de la educación pública, ya que garantiza la exoneración impositiva de aquellas instituciones de enseñanza privada. En base al sistema de organización unitario, la cuestión educativa no escapa de la órbita nacional, y son prácticamente nulas las facultades delegadas en la organización departamental (Alvarez et al, 2020). El artículo 275 inciso $9^{\circ}$ de la Constitución de la República se limita a poner en tarea de los intendentes la custodia de la educación, y un derecho de voz frente a las autoridades educativas competentes. Por otra parte, se encuentran garantizadas la libertad de enseñanza de los padres hacia los hijos, con una intervención mínima del Estado «al solo objeto de mantener la higiene, la moralidad, la seguridad y el orden públicos» (artículo 68). La legislación aprobada en 2008 establece las regulaciones contemporáneas de este derecho.

\section{Discusión: elucidaciones sobre un labora- torio normativo}

El análisis comparativo entre los textos constitucionales de los países considerados evidencia que el derecho a la educación concita una marcada atención por la cual se establecen una serie de obligaciones estatales para reconocerlo y garantizarlo. Por ello, es posible afirmar que en el nivel normativo constitucional -en los países seleccionados - ha existido un pronunciado consenso en relación con el reconocimiento de este derecho. Lo cual evidencia la diseminación de mecanismos de armonización (Dale, 1999) y que permite concebir incluso un potencial laboratorio normativo debido a la profusión de disposiciones constitucionales sobre la educación como derecho en estos países emergentes.

Las Constituciones nacionales, sobre todo la de Argentina y Brasil -ambos países con regímenes federales de gobierno-, poseen numerosos artículos en los que, como en el caso de la educación, se procura detallar exhaustivamente el alcance del derecho, las obligaciones del Estado, los objetivos, los fines, entre otros aspectos. Pese a lo ambiciosas que puedan parecer sus cláusulas, ellas constituyen una importante apuesta para avanzar en el reconocimiento y en la protección de los derechos individuales y colectivos, y así favorecer un acceso a la justicia más extendido y la promoción de un sistema judicial más permeable a las demandas sociales (Gargarella, 2008). A pesar de que en todos ellos se reconocen una serie de contenidos básicos similares, el desarrollo normativo legal que se deriva de estas Constituciones, así como los resultados de las políticas en que se fundan (lo cual excede los alcances de este estudio), presentan resultados con importantes diferencias dentro del propio MERCOSUR (que constituye el criterio de demarcación de los países aquí estudiados). Ello se advierte en las bases legales de la educación, en la organización sistémica de la escolarización y en la distribución de competencias entre los diferentes niveles de gobierno en cada uno de estos países. 
A partir de este encuadre, es posible establecer algunas caracterizaciones derivadas del análisis del derecho a la educación en estos países considerados. En todos ellos existe un reconocimiento de este derecho en las bases constitucionales y sus alcances se explican en relación con los cambios ocurridos en varios países latinoamericanos, y que tuvieron impacto directa o indirectamente en lo educativo. Particularmente, en muchos países sudamericanos ha existido un consenso en relación con el reconocimiento de los derechos sociales en el conjunto global de las Constituciones en el nivel regional. En tal sentido, se destacan las reformas constitucionales de Colombia (1991), Paraguay (1992), Argentina (1994), Ecuador (1998 y 2007-2008), Venezuela (1999) y Bolivia (2006-2009). Estas reformas se explican -en algunos casos- como reacción a las políticas neoliberales de la década de 1990, y además poseen valores y prácticas impulsadas por un modelo constitucionalista más radical que supera el nominalismo institucional presente en las Constituciones más formales (Viciano y Martínez, 2010). En muchas de esas políticas de reformas del Estado, la educación escolar ha sido objeto de un conjunto de medidas que favorecieron su redefinición no como derecho sino como un bien de mercado, o como un bien público. ${ }^{6}$

Sin embargo, se debe advertir que en estos cuatro países, los sistemas escolares han sido objeto de reformas escolares que implicaron cambios notorios en términos de extensión de la obligatoriedad de los estudios, definiciones curriculares, y en muchos casos favorecieron durante las últimas décadas del siglo pasado procesos de desregulación de la educación privada así como de descentralización escolar, los cuales se enmarcaron en programas de ajuste del Estado con matices en cada caso nacional. En particular se destaca el caso de Chile por las políticas de profunda privatización del sistema educativo a partir de la década de 1980 (Santa Cruz Grao y Olmedo, 2012; Verger, Bonal y Zancajo, 2016). En muchas de esas políticas se desplegaron medidas que favorecieron la redefinición de la educación escolar y no necesariamente en atención al concepto de derecho humano. Más aun, diferentes estudios en la región han destacado cómo los gobiernos de algunos países sudamericanos han arremetido contra la educación pública con políticas que afectaron las finalidades de la escuela púbica, así como sus prácticas, y con ello han perjudicado el ejercicio del derecho a la educación por parte de sus titulares (Frigerio, 2000; Gajardo, 1999; Kaufman y Nelson, 2005; Vior et al., 2016). En diferentes países la desregulación de la educación privada fue instrumentada como respuesta a la ineficacia y a la ineficiencia de lo público denunciadas por los discursos favorables a las reformas. Lo cual es particularmente evidente en el caso chileno (Santa Cruz Grao y Olmedo, 2012; Verger et al., 2016).

No obstante, es evidente que estas Constituciones sudamericanas reformadas, además de garantizar un control real del poder por parte de los ciudadanos, buscan responder a

$6 \quad$ El concepto de bien público no posee una definición unívoca. Podría sostenerse que es un bien el que genera beneficios o utilidades apropiables conjuntamente por la comunidad en forma grupal. Aunque el beneficio no es para todos igual, sí lo es el hecho de que nadie queda excluido de su consumo o uso: el consumo está garantizado para todas las personas. Desde la perspectiva de algunos organismos internacionales de financiamiento, la educación básica integra el listado de bienes públicos (Ruiz, 2020).

Por otra parte, pensar a la educación como bien de mercado implica definirla como una mercancía. Todos los bienes de mercado se caracterizan porque generan beneficios o utilidades que pueden ser apropiadas exclusivamente por el individuo que los consume; son bienes que se ajustan al principio de exclusión, es decir, resulta posible excluir del consumo a quien no haya relevado sus preferencias en el mercado, por no ofrecer pagar un precio por ello. En algunas reformas de la derecha moderna, como las británicas instrumentadas a partir de la década de 1980, se perciben estas orientaciones para la educación (Whitty et al, 1999). 
la pregunta de cómo solucionar el problema de la desigualdad social (Scioscioli y Ruiz, 2020). En tal sentido, en primer lugar, todas ellas incluyen en sus cláusulas instancias participativas y establecen compromisos sociales específicos. Son textos constitucionales con numerosos artículos en los que, como ocurre en el caso de la educación, se procura detallar exhaustivamente el alcance del derecho, las obligaciones del Estado, los objetivos, los fines, el financiamiento, la administración y el gobierno, entre otros aspectos. Es aquí, en esta similitud en las disposiciones constitucionales, que se observa cierto proceso de armonización (Dale, 1999) en relación con el avance de posturas y definiciones propias de las técnicas del constitucionalismo internacionalizado. ${ }^{7}$ Pese a lo ambiciosas que puedan parecer sus cláusulas (que permiten plantear la conjetura de un laboratorio normativo), ellas constituyen una importante apuesta para avanzar en el reconocimiento y en la protección de los derechos individuales y colectivos, y así favorecer un acceso a la justicia más extendido, una mayor integración social, la promoción de un sistema judicial más permeable a las demandas sociales (Gargarella, 2008), y la posibilidad de que las decisiones judiciales puedan influir en la implementación de los programas gubernamentales. En segundo lugar, la mayoría de las Constituciones estudiadas ha dado lugar al reconocimiento de la diversidad cultural de los pueblos, especialmente de los originarios (Ruiz, 2020). De todos modos, se destacan diferencias en los períodos en los cuales han entrado en vigor estas Constituciones analizadas: mientras que en Uruguay y en Chile se encuentran textos constitucionales de décadas pasadas, en Brasil y Argentina las Constituciones son más recientes y receptan muchas de las instituciones derivadas del constitucionalismo internacionalizado. A pesar de ello, en todos los casos el derecho a la educación es reconocido y garantizado por estas Constituciones y es definido como un derecho humano.

Así, se observa que en Uruguay la educación se apoya en los principios explicitados en la Constitución de la República (1967) y en la normativa aprobada en el año 2008 que recogen principios que dan contenido al derecho a la educación en su país, tales como la libertad de pensamiento, de enseñanza, la laicidad, la obligatoriedad, la gratuidad y el principio de autonomía de la enseñanza con respecto al Poder Ejecutivo Nacional. En su artículo 68 la norma dispone que «queda garantida la libertad de enseñanza» y que una ley «reglamentará la intervención del Estado al solo objeto de mantener la higiene, la moralidad, la seguridad y el orden públicos». Con respecto a los niños, se establece que los padres y tutores tienen el derecho de elegir para la educación de sus hijos o pupilos los maestros o instituciones que deseen. Si bien el Estado es laico, no se prohíbe la enseñanza religiosa. Se fija también la obligatoriedad de la enseñanza primaria y media, agraria o industrial. En el artículo 71 se declara «de utilidad social la gratuidad de la enseñanza oficial primaria, media, superior, industrial y artística y la educación física [...] y el establecimiento de bibliotecas populares».

$7 \quad$ Se denomina de esta forma a uno de movimientos del constitucionalismo, surgido luego del año 1945 (Elster, 1998). Desde esta perspectiva, para la protección de los derechos no basta con el reconocimiento de derechos y garantías (como ocurre en el constitucionalismo clásico y en el social) se requiere además el establecimiento de obligaciones estatales (de respetar, garantizar, promover y facilitar) para el ejercicio de los derechos de los habitantes en el ámbito nacional e internacional. Este constitucionalismo internacionalizado se nutre de diversas técnicas, entre ellas se destacan: el establecimiento de un estatus privilegiado a las normas sobre derechos humanos; el reconocimiento de la competencia de organismos internacionales de protección de los derechos humanos para que el Estado pueda ser juzgado en esas jurisdicciones internacionales; la relevancia de la jurisprudencia producida por esos organismos internacionales para la aplicación e interpretación de las normas de derechos humanos; la imprescriptibilidad de los delitos de lesa humanidad; la ampliación de los derechos sociales; el reconocimiento de derechos de incidencia colectiva. 
En la Constitución Federal de Brasil (1988), por su parte, el derecho a la educación ha sido objeto de una regulación extensa por la cual varios artículos refieren a aspectos tales como el acceso a la educación, su calidad y el financiamiento, la asignación de responsabilidades, la distribución de funciones entre los entes de la federación, la organización del sistema educacional. Se destaca lo dispuesto en el artículo 205 que fija que la educación es un «derecho de todos y un deber del Estado y de la familia». Esto último se encuentra reforzado en el artículo 227 en el que se afirma que «es deber de la familia, de la sociedad y del Estado, asegurar al niño, al adolescente y al joven, con absoluta prioridad, el derecho a [...] la educación». La norma constitucional también prevé principios vinculados con la igualdad de condiciones para el acceso y la permanencia en la escuela, el pluralismo de concepciones pedagógicas, la gratuidad de la enseñanza pública y la garantía de un estándar de calidad, entre otros. La Constitución garantiza asimismo la educación básica obligatoria y gratuita desde los 4 a los 17 años, la atención educativa especializada para personas con discapacidades y la educación infantil hasta los 5 años.

En el caso de la Argentina el derecho a la educación ha obtenido un amplio marco de reconocimiento constitucional con especial énfasis en la protección de este derecho durante la niñez. Se reconoce el «derecho de enseñar y aprender» a todos los habitantes (artículo 14), y con la incorporación de los instrumentos internacionales de derechos humanos, «en las condiciones de su vigencia» en su artículo 75 inciso 22 se termina por definir a la educación como un derecho humano al que se adosa un piso de contenidos que forman parte de las obligaciones internacionales del Estado argentino en esta materia. La Constitución también prevé la responsabilidad indelegable del Estado en educación, la que cobra sentido con la gratuidad de la educación pública. Se reconoce expresamente a la niñez como grupo vulnerable, lo cual deriva en la obligación del Estado de establecer medidas de acción positivas que garanticen la igualdad real y el pleno goce de sus derechos (artículo 75 inciso 23).

Finalmente, entre los países analizados se distingue el caso de Chile dado que su Constitución fue aprobada en 1980 -durante una dictadura militar- a pesar de que el texto constitucional tuvo varias reformas en los años posteriores. En su artículo 19 se define a la educación como un derecho humano (inciso 10) pero a la vez se otorga especial centralidad a la libertad de enseñanza (inciso 11). Los aspectos más notorios en cuanto a la definición de los alcances del derecho a la educación en este país están dados por su legislación, tanto la aprobada durante el período del gobierno militar cuanto por la sancionada durante los gobiernos democráticos. Sobresalen no solo las limitaciones en la cuestión de la gratuidad de los estudios en el nivel superior, sino además una clara desregulación de la educación privada que se ha visto favorecida por una normativa que les permite el acceso a fondos públicos para su desarrollo. Asimismo, las obligaciones estatales en materia del derecho a la educación no aparecen tan claramente marcadas por el texto constitucional como en los demás países aquí analizados.

Para concluir este estudio se podrían destacar las dificultades que presenta la definición conceptual unívoca del derecho a la educación a la luz de las cláusulas constitucionales; quizás en parte se deba a que la educación como práctica es culturalmente diversa y como derecho es históricamente variable. Incluso si se lo define desde los estándares normativos internacionales, relativos a las obligaciones de los Estados, es muy difícil encontrar correspondencias estrictas entre los casos nacionales. Más difícil resulta aún garantizar el ejercicio de este derecho en el plano de las políticas, de las instituciones y de los actores locales. La falta de precisión conceptual y el desconocimiento sobre las 
implicancias como derecho humano a su vez redunda en la vaguedad argumentativa con la cual se invoca desde diferentes ámbitos este derecho.

Sin embargo, a partir de la conceptualización de la educación como derecho fundamental es posible comprender su carácter de derecho humano como derecho universal reconocido por igual a todas las personas en su calidad de tales (Scioscioli, 2015). Se trata de un derecho que se asienta en una misma base moral de fundamentación basada en la dignidad de la persona, de su valor en cuanto a fin en sí misma. Dicha base se encuentra establecida como punto de partida y de respeto, y ha sido operacionalizada en los principales instrumentos internacionales de derechos humanos a partir de la Declaración Universal aprobada en 1948. De acuerdo con el enfoque de Dale (1999), este desarrollo normativo daría cuenta de un proceso de armonización en lo referido a las definiciones sobre el derecho a la educación. Por ello, se puede sostener que en el plano de las normas (supralegales) se evidencian acuerdos internacionales que demuestran este potencial para concebir un laboratorio normativo en esta región.

En suma, es posible sostener que, a pesar de la ausencia de una definición constitucional unívoca sobre el contenido del derecho a la educación, en el plano de las normas se evidencian algunos de los acuerdos internacionales que se plasmaron en las normativas locales y que reflejan los compromisos de los instrumentos internacionales de derechos humanos. Todo ello ha reconfigurado el derecho a la educación, y incluso ha dado fundamento para considerarlo un derecho de incidencia colectiva. No obstante, pese a que en la mayoría de las Constituciones estudiadas se reconocen una serie de contenidos básicos similares, cabría completar y profundizar el estudio con el análisis del marco normativo legal que se deriva de los textos constitucionales, así como en los efectos de las políticas públicas instrumentadas. Es allí donde se observan resultados empíricos con importantes diferencias en los contextos nacionales aquí considerados. Ello afecta el ejercicio de este derecho por parte de la población, y pone en evidencia las diferencias entre el plano de las normas y el plano de las acciones de los Estados referidas al derecho a la educación, el más igualador de los derechos humanos fundamentales.

\section{Referencias bibliográficas}

Alexy, R. (2008). Teoría de los derechos fundamentales. Madrid: Centro de Estudios Políticos y Constitucionales.

Alvarez, G., Bugay, A., Crolla, M., Tagliani, F. y Yaber, T. (2020). El derecho a la educación según las bases constitucionales y legales en los países de América del Sur. En G. Ruiz (dir.), El derecho a la educación: definiciones, normativas y políticas públicas revisadas (pp. 65-111). Buenos Aires: EUDEBA.

Biasco, E. (2001). La gratuidad de la enseñanza pública en el nivel superior. Montevideo: ProEVA-UDELAR.

Broadfoot, P. (2000). Comparative education for the 21st century: retrospect and prospect. Comparative Education, 36(3), pp. 357-372.

Clérico, L. (2009). El examen de proporcionalidad en el derecho constitucional. Buenos Aires: EUDEBA. 
Cury, C. R. J. (2016). Vinte Anos da Lei de Diretrizes e Bases da Educação Nacional (LDBEN). Jornal de Políticas Educacionais, 10(20), 3-17.

Dale, R. (1999). Specifying globalization effects on national policy: A focus on Mechanisms. Journal Education Policy, 1(14): 1-17.

Elster, J. (1998). Ulises revisitado. Compromisos previos y constitucionalismo. Análisis político, 35, 62-85.

Ferrajoli, L. (2016). Los derechos y sus garantías. Conversación con Mauro Barberis. Madrid: Trotta.

Ferraro, A. R. (1985). Analfabetismo no Brasil: tendencia secular e avanços recentes. Resultados preliminares. Cadernos de Pesquisa, 52, 35-49.

Frigerio, G. (2000). ¿Las reformas educativas reforman las escuelas o las escuelas reforman las reformas? Seminario sobre Prospectivas de la Educación en América Latina y el Caribe. Santiago de Chile.

Gajardo, M. (1999). Reformas educativas en América Latina. Balance de una década. Santiago de Chile: PREAL.

Gargarella, R. (coord.) (2008). Teoría y crítica del derecho constitucional. Buenos Aires: Abeledo Perrot.

Grupo de Desarrollo Sustentable de las Naciones Unidas (2003). Declaración del Taller interinstitucional de Stamford sobre el "entendimiento común" del enfoque de la cooperación para el desarrollo basado en los derechos humanos. s/d

Kaufman, R. y Nelson, J. (2005). Políticas de Reforma Educativa. Comparación entre países. Santiago de Chile: PREAL.

Lawn, M. (2013). A Systemless System: designing the disarticulation of English State Education. European Educational Research Journal, 12(2), 447-462. Recuperado de https://journals.sagepub.com/doi/10.2304/eerj.2013.12.4.447

Lubienski, C. (2006). School Choice and Privatization in Education: An Alternative Analytical Framework. Journal for Critical Education Policy Studies, 4 (1). Recuperado de http://www.jceps.com/wp-content/uploads/PDFs/04-01-10.pdf

Oficina del Alto Comisionado para los Derechos Humanos de las Naciones Unidas (2006). Preguntas frecuentes sobre el enfoque de derechos humanos en la cooperación para el desarrollo. New York: UUNN.

Olmedo, A., Bailey, P. y Ball, S. (2013). To Infinity and Beyond...: Heterarchical Governance, the Teach For All network in Europe and the making of profits and minds. European Educational Research Journal, 12(4), 492-512. Recuperado de http://dx.doi.org/10.2304/eerj.2013.12.4.492

Ruiz, G. R. (2020). El derecho a la educación: definiciones, normativas y políticas públicas revisadas. Buenos Aires: EUDEBA. 
Schulte, B. (2016). La teoría de la cultura global con características chinas: cuando los modelos globales se tornan nativos. En G. Ruiz y F. Acosta (eds.), Repensando la Educación Comparada. Lecturas desde Iberoamérica. Entre los viajeros del siglo XIX y la globalización (pp. 99-116). Barcelona: Octaedro.

Scioscioli, S. (2015). La educación básica como derecho fundamental. Implicancias y alcances en el contexto de un Estado Federal. Buenos Aires: EUDEBA.

Scioscioli, S. y Ruiz, G. R. (2020). Droit constitutionnel et droits des enfants en Amérique latine. A. Martin (Ed.), L'enfance et le droit en Amérique latine (pp. 147-187). L'Harmattan.

Santa Cruz Grao, E. y Olmedo, A. (2012). Neoliberalismo y creación de 'sentido común': crisis educativa y medios de comunicación en Chile. Profesorado. Revista de curriculum y formación de profesorado, 16(3), 145-168. Recuperado de http:// www.ugr.es/local/recfpro/rev162ART8.pdf

Schriewer, J. (2010). Comparación y explicación entre causalidad y complejidad. En J. Schriewer y H. Kaelble (eds.), La comparación en las ciencias sociales e históricas (pp. 17-62). Barcelona: Octaedro.

Steiner-Khamsi, G. (2016). La transferencia de políticas como herramienta para comprender la lógica de los sistemas educativos. En G. Ruiz y F. Acosta (eds.), Repensando la Educación Comparada. Lecturas desde Iberoamérica. Entre los viajeros del siglo XIX y la globalización (pp. 27-40). Barcelona: Octaedro.

Steiner-Khamsi, G. (2010). The Politics and Economics of Comparison. Comparative Education Review 54(3), 323-342.

Tomasevski K. (2001). Human rights obligations: making education available, accessible, acceptable and adaptable. Gothenburg: Novum Grafiska AB.

Verger, A., Bonal, X. y Zancajo, A. (2016). What Are the Role and Impact of PublicPrivate Partnerships in Education? A Realist Evaluation of the Chilean Education Quasi-Market. Comparative Education Review, 6o(2), 223-248.

Viciano Pastor, R y Martínez Dalmau, R. ¿Se puede hablar de un nuevo constitucionalismo latinoamericano como corriente doctrinal sistematizada? Trabajo presentado en el VIII World Congress of International Association of Constitutional Law, México, 6-10 de diciembre de 2010. Recuperado de www.juridicas.unam.mx/wccl/ ponencias/13/245.pdf

Vior, S., Rodríguez, L. y Más Rocha, S. M. (2016). Evaluación, calidad y escuela secundaria en la Ciudad de Buenos Aires (1992-2013). Luján: EdUnLu.

Whitty, G. Power. S. y Halpin, D. (1999). La escuela, el Estado y el mercado. Delegación de poderes y elección en educación. Madrid: Morata. 